\title{
Superconducting Cavities Automatic Loaded Quality Factor Control at FLASH
}

\author{
W. Cichalewski, J. Branlard, H. Schlarb, J. Carwardine, A. Napieralski
}

\begin{abstract}
The well-established technology of superconducting niobium cavities (TESLA) finds an increasing number of applications for linear accelerators in high-energy physics experiments. Together with cavity design and manufacturing, control systems for accelerating field parameters were developed. The digital control system developed for cryomodule operation at the Free Electron Laser in Hamburg (FLASH) is able to perform field amplitude and phase regulation with the precision requested for the multiple user experiments carried at FLASH and according to the required laser light parameters. While new experiments (like the International Linear Collider or the proposed European $X$-FEL upgrades) are based on the same technology (and similar control systems), they require additional levels of controlling superconducting structures parameters in order to comply with tighter power budget overheads and finer field regulation requirements. Consequently, the possibility of controlling such parameters as cavity loaded quality factor or resonance frequency are becoming more attractive. The necessity and the benefits of regulating these parameters has been described in [1] and [2].
\end{abstract}

\section{INTRODUCTION AND MOTIVATION}

$\mathbf{S}$ uperconducting cavities in Free Electron Laser (FEL) experiments are used to accelerate the electron beam in presence of electromagnetic field. Standing wave generated in the structure provide the optimal environment for particle acceleration as long as the field parameters are controled with a precision that assures stability of about $10^{-4} \mathrm{dA} / \mathrm{A}$ and $0.01 \mathrm{deg}$ (depending on the requested beam energy) for amplitude and phase respectively. Typically, these requirements are defined for the control loop regulation which includes 6 to 8 cavities (i.e. one cryomodule). Due to cost effectiveness, many FEL experiments are using one microwave amplifier to supply power to one or more cryomodules. A vector-sum based control approach [5] is then used for fast and easy compensation of low performance superconducting structure. On the other hand, more cavities in the overall vector implies lower controller impact on each structure. Fortunately, in the TESLA technology module design, other indirect regulators allow the system user to shape some specific resonator parameters in the loop. For example, control over the mechanical frequency tuners and input couplers also give the possiblity for a more adequate and precise management of the required power budget. Each TESLA cavity is equipped

W. Cichalewski and A. Napieralski are with the Department of Microelectronics and Computer Science - Technical University of Lodz, ul. Wolczanska 221/223 Lodz, 90-924 Poland (telephone: +48 42 6312720, email: wcichal@dmcs.pl).

J. Branlard and H. Schlarb are with the Deutsches Electronen Synchrotron, Notkestrasse 85 Hamburg, 22-607 Germany (telephone: +49 4089981599 , e-mail: julien.branlard@desy.de).

J. Carwardine is with Argonne National Laboratory, 9700 South Cass Avenue Lemont, IL 60439, USA

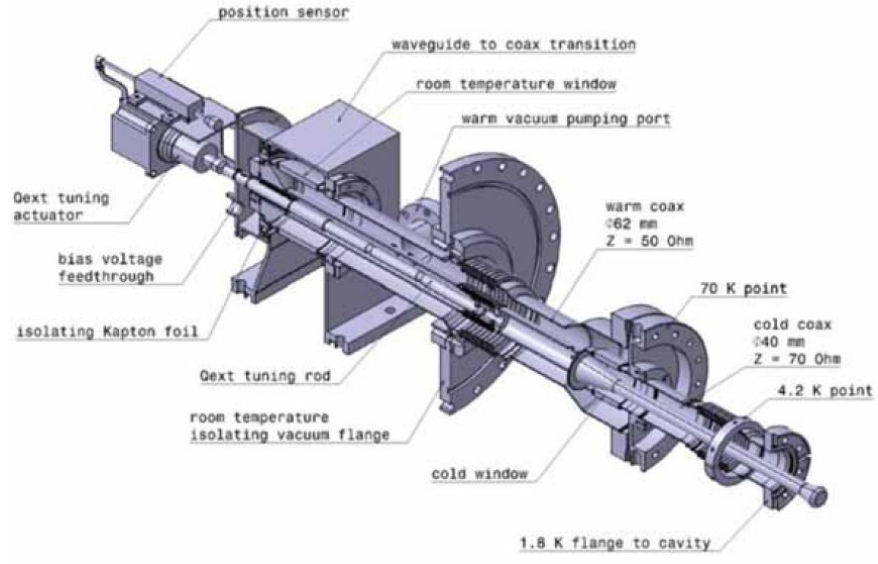

Fig. 1. Structure of cavity input coupler for X-FEL modules [3]

with a fundamental power coupler (FPC), illustrated in Fig. 1). Inside, an antenna couples the RF power signal (typically, pulsed with a duration of $1.3 \mathrm{~ms}$, a frequency of $1.3 \mathrm{GHz}$, a repetition rate of $10 \mathrm{~Hz}$ and a power of 200-300 $\mathrm{kW}$ ) into the resonator resulting in electro-magnetic fields in which electron bunches are accelerated. In a similar way the slow and fast frequency tuners can maintain each cavity to its resonance frequency [4], the motorized FPC can act on the effective cavity bandwidth, hence modifying the transient response of the resonator during the acceleration process, which is the most important from the point of view of energy transfer to the electron beam. Typical loaded quality factor $(\mathrm{Ql})$ values for superconducting high-Q resonators is on the level of $3 \mathrm{e} 6$. Fundamental pulse operation parameters such as the fill time and profile are based on this value and are calculated for the whole module or RF station. The measured RF field for individual cavities may deviate from the vector sum request or set-point, even if the vector regulation criteria are fulfilled. This can be due to discrepancies among cavity performances (maximal operational gradient or quench limit), different levels of frequency detuning, unequal power distribution among the structures in one module or also due to thermal effects in each FPC. By adjusting its quality factor, it is possible to optimize the cavity power filling profile. In practice, a better control on the field distribution for individual cavities allows for a more efficient power budget usage and operation closer to the performance limit. 


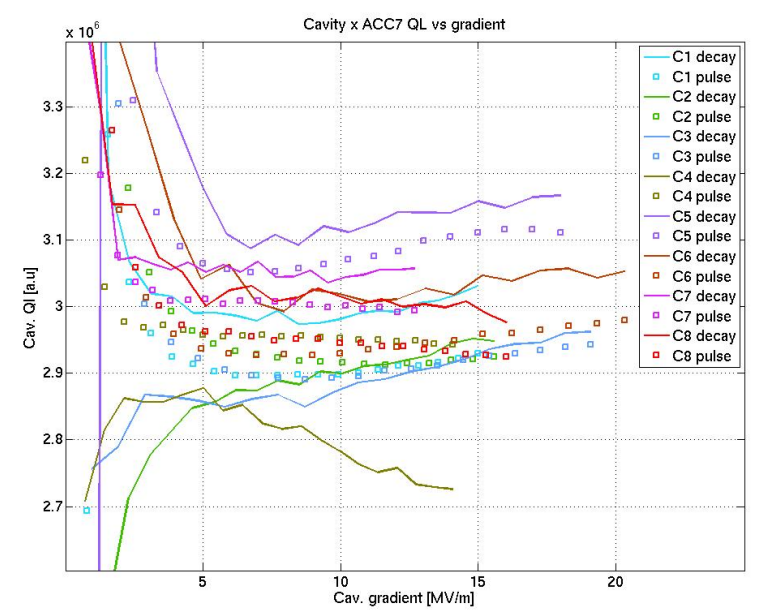

Fig. 2. Q1 for all cavities in one cryomodule (ACC7 at FLASH) as a function of the cavity field gradient.

\section{Algorithm Design}

The design of the Q1 control algorithm relies on accurate monitoring of cavity parameters such as operating gradient and phase, quality factor and detuning. These parameters can be measured from the time response waveforms.

\section{A. Quality Factor Measurement}

The cavity external quality factor Qext, can be adjusted using the fundamental input cavity coupler. The change of the input power coupling to the resonator modifies the external Q, hence changing the energy storing ability of the cavity during the RF pulse:

$$
\frac{1}{Q l}=\frac{1}{Q e x t}+\frac{1}{Q o}
$$

where Q1, Qext and Qo are the loaded, external and unloaded quality factors, respectively. The cavity detuning and loaded quality factor are determined during the on-line pulse to pulse data analysis, based on the second-order resonator state-space model response [5]. An alternative approach to computing Q1 is done using the cavity response during the decay of the RF pulse. A comparison of the two methods, calculated for all 8 cavities in one cryomodule module at FLASH is illustrated in Fig. 2.

The Q1 tuning algorithm can work in a reliable way only if the dependency between measured Q1 values and cavity gradient is well understood and characterized. It was observed that in the case of high Q resonators, the measured Q1 for highly detuned cavities can differ significantly from its onresonance value. On a first order, we can assume that the frequency deviation is monitored and compensated for by a piezo based system, responsible for maintaining the cavities on resonance [4].

\section{B. Quality Factor - FPC Motor Position Characterization}

In today's TESLA technology, the FPC position can be adjusted by a step motor, connected to the external tip of the

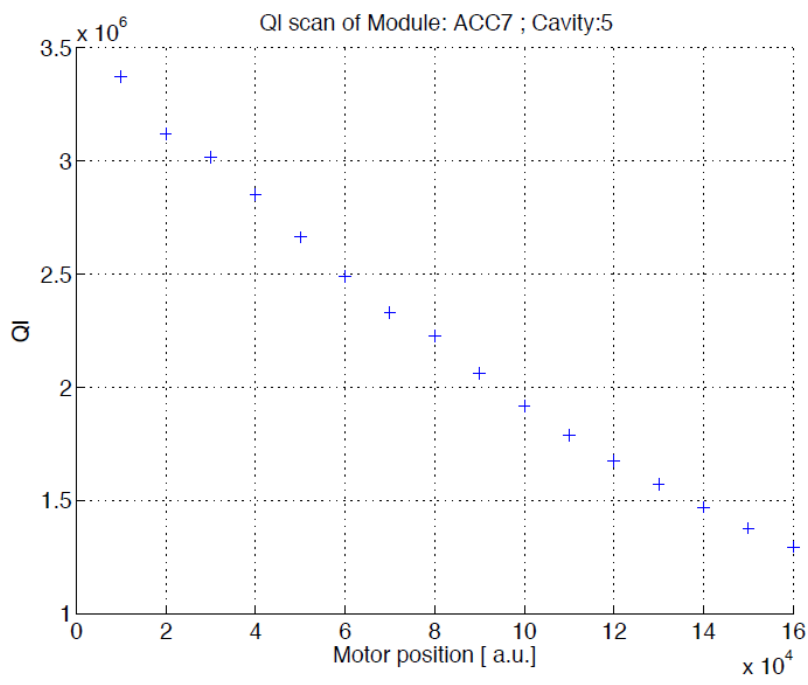

Fig. 3. Example of input coupler motor transfer function measured for one cavity.

power coupling antenna. A typical transfer function characterizing the cavity $\mathrm{Ql}$ as a function of its step motor position is illustrated in Fig. 3. This transfer function is measured in order to provide accurate control of the structure quality factor. The characterization has been performed for each superconducting structure from accelerating modules 6 and 7 at FLASH. A nearly linear dependency between $\mathrm{Ql}$ and the motor position can be seen on Fig. 3. However, this relation becomes highly nonlinear outside of the nominal 3e6 cavity Q1 setting. This characterization also allows to determine the maximum Q1 range with the existing set-up. These measurements will be automated for the cryomodule tests planned at the Accelerating Module Test Facility (AMTF) during the installation phase of the European XFEL [6].

\section{Proportional Feedback Control}

For each cavity, the control algorithm calculates the error between the measured factor averaged over a given number of measurements and the requested set-point value:

$$
Q l_{\text {error }}=Q l_{s p}-Q l_{\text {measured }}
$$

This error is scaled by the input antenna motor constant, $M_{\text {const }}$ (calculated during the motor characterization studies) and a proportional controller gain $K_{P}$ is applied. A new step motor position is then calculated and the device is moved by the required amount of steps.

$$
M_{\text {newpos }}=M_{\text {currentpos }}+M_{\text {const }} \times Q l_{\text {error }} \times K_{P}
$$

where $M_{\text {currentpos }}$ and $M_{\text {newpos }}$ are the current and new motor position, respectively. As identified during the step motor characterization, a change of motor position corresponds to a linear change of Q1. This relationship is used to determine the initial motor constant value. This initial value can then be dynamically modified and monitored for control loop exception handling. The proportional gain coefficient $K_{P}$ has to be chosen in a way not to cause loop divergence. This 


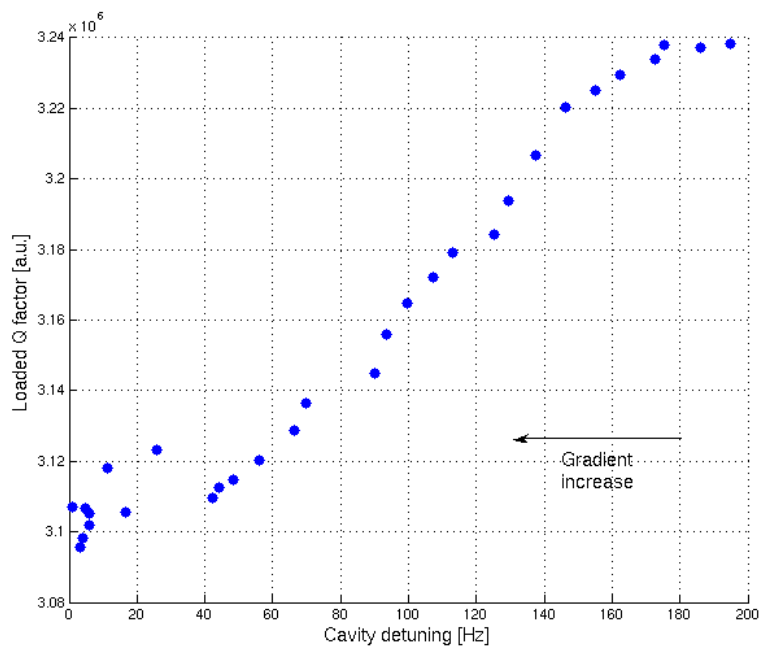

Fig. 4. Q1 in function of the cavity detuning (for different field gradient)

parameter is usually chosen between 0 and 1 . Its value has a direct impact on the algorithm regulation speed.

\section{Algorithm and System Limitations}

Three sources of limitations have been identified during the system behavior study. These exceptions can potentially compromise the performance of the algorithm described above and prevent it from bringing the cavity Q1 factor to its desired set-point:

1. Motor in end-switch position: it can happen that the required set-point is outside the coupler regulation range. In this case, the algorithm should ignore the requested quality value,

2. The cavity frequency detuning distorts Q1 measurement: it has been verified that the measured factor value is changing with cavity detuning. In such a case, the algorithm will try to bring the cavity to its set point based on distorted Q1 measurements which may result in extensive motor excursions,

3. Low cavity gradients prevent reliable Q1 measurements: a strong dependence of the Q1 measurement on the cavity gradient has been observed and is illustrated in Figure 4. To cope with this issue, the algorithm initially checks the present cavity gradient and prevents coupler motor rotations should the measured gradient be lower than its acceptable threshold.

In order to minimize the risk associated with the issues mentioned earlier, two mechanisms have been implemented in order to:

1. check if the algorithm is not diverging. A step-to-step increase in the $\mathrm{Ql}$ error is detected as a divergence and is reported. A constant Q1 error increase results in algorithm termination,

2. evaluate if the motor is still moving while the antenna position is not changing. If the calculated motor constant is below a certain threshold (for several algorithm iterations), it means the coupler antenna is stuck and moving the motor does not result in a change in loaded Q. Such a situation also results in algorithm termination.

Some of the issues mentioned above can be minimized by improving the existing acquisition system. The uTCAbased LLRF system [7] upgrade at FLASH scheduled for this summer offers better signal resolution, higher sampling rate and flexibility in the acquisition range optimization which have a significant influence during cavity parameters identification.

\section{Automatic Ql Control Implementation}

Typical modifications of cavity loaded $Q$ values are either due to a fundamental change of the experiment settings, or due to slow temperature related drifts. In both cases, any Q1 setting change is not required at the pulse repetition rate level $(10 \mathrm{~Hz})$. Hence, the design of the described algorithm does not require either fast loop actuators or loop controller response response in the micro-seconds level. For this reason, the Q1 control algorithm was implemented as a high-level software component.

\section{A. Functional Software Structure}

The algorithm was prepared as an application-level server, compatible with the DOOCS (Distributed Object Oriented Control System) at DESY [8]. The middle-layer server which runs this functionality has been added as a permanent element of control tools used in accelerator of FLASH. This Q1 adjustment server needs to communicate with control system components in order to:

1. Acquire the system current status. This is realized by communication with the system middle-layer diagnostic server that provides information about individual resonator (acquiring loaded Q values, cavity gradients, detuning information),

2. Acquire the actuator status and manage motor position change. This part is provided by the step motor front-end server that communicates with the device controller,

3. Monitor system error status - the information about cavity quench is used to terminate any algorithm activity. This knowledge is gained from the quench detection middlelayer server.

The main scenario of the application work consists of several steps that are performed each time the application is triggered (see Fig. 5). The algorithm is triggered at a $1 \mathrm{~Hz}$ rate for Q1 data analysis, motor status verification and/or motor position change request.

Each run of the algorithm starts from the evaluation of the connection to external servers front-end and middle layer servers required for the error signal calculation and motor management. When all the needed data is available, the application looks for user permission to proceed. If it is granted, the validity of the measured Q1 value is checked, based on the information about current gradient level and the measured cavity detuning. If all conditions are fulfilled, the next step is to follow one of the scenario paths based on the current actuator (step motor) status. In case the motor is in "busy", "error" or "end-switch" state, the corresponding 


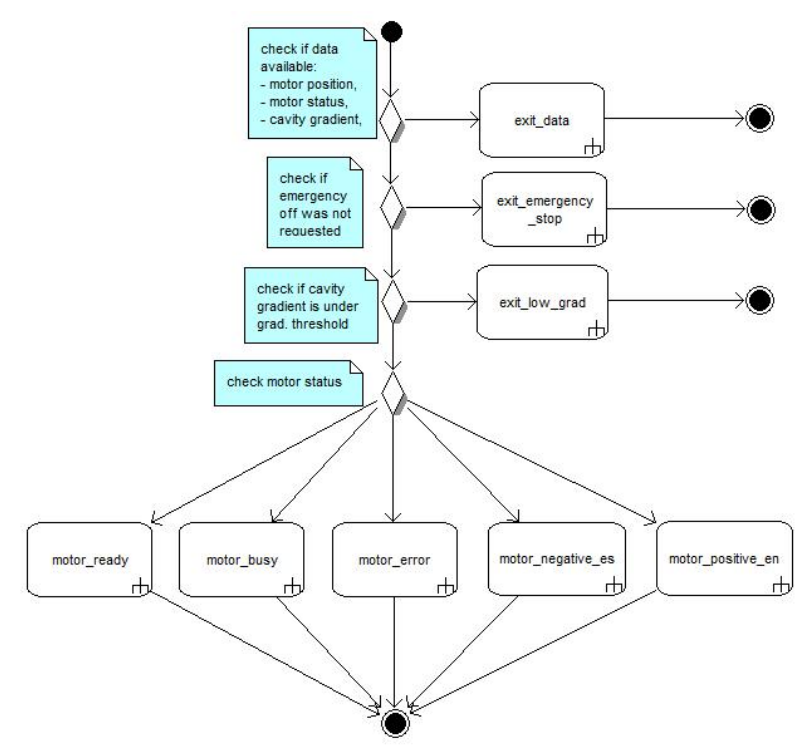

Fig. 5. Server main activity diagram

exception handling procedure is executed. If the system cannot be brought to an operational state, the error status is reported and the application terminates the in-loop Q1 correction. On the other hand, the current loop controller part is triggered (see Fig. 6) as long as the "ready" state is maintained.

If the motor status allows for loaded Q adjustment, the application acquires the measured Q1 value and the current motor position. Based on the set-point information, the new FPC antenna position is determined. If the calculated position change exceeds a pre-defined threshold, its excursion is truncated. Then, the algorithm checks whether the new position is within the motor regulation range (inside software endswitches). Before the server sends the request to move the motor to its new setting, some additional safety conditions are checked. The algorithm checks whether:

1. the algorithm is not diverging. In case of faulty motor performance or wrong loop parameter adjustments, an increase of the measured deviation from the set-point is detected. If such a situation remains for a pre-defined time period, the algorithm stops its operation and reports a loop diverging exception to the user,

2. the motor position change no longer result in a $\mathrm{Ql}$ change. The motor constant factor is constantly monitored. If the motor constant value decreases and the motor spinning is no longer effective, the algorithm terminates its task and informs the user about the encountered problem,

3. the motor position change is within regulation accuracy range. In case the calculated new motor position has no net effect on the quality factor value, the actuator is not moved.

\section{B. Operator Interface}

In order to provide easy and efficient interface for the application parameters configuration (expert - cavity panel) and visualization of tool activity results, a set of jDDDbased panels (java Doocs Data Display [9] was prepared. The

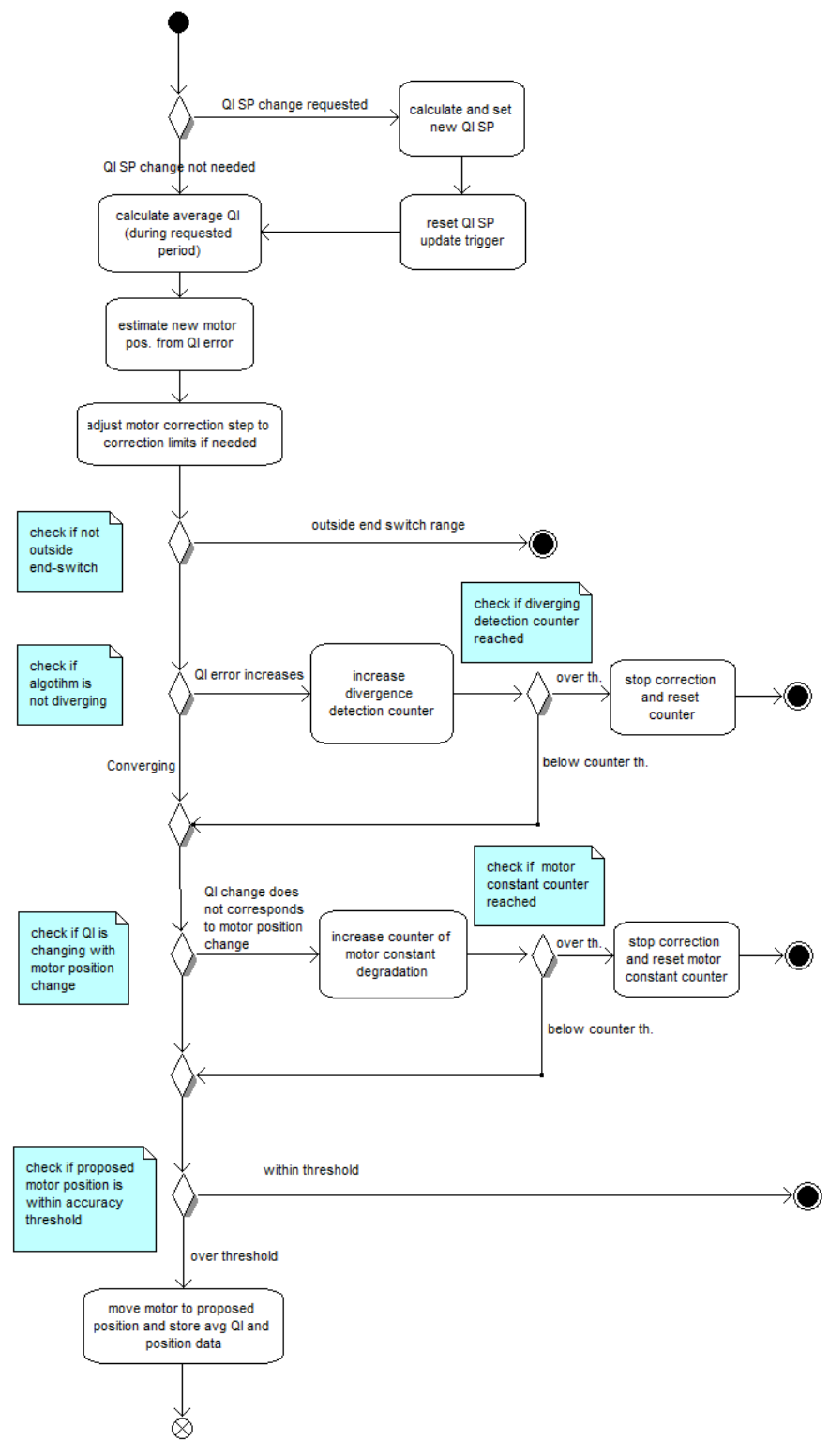

Fig. 6. Check and set activity diagram

general part of the GUI includes main information about the algorithm and each cavity status as depicted in Fig. III-B). Knobs for algorithm enabling/disabling, required Q1 settings, current measured quality factor, current motor position and user messages are placed on this window.

More expert related parameters can be found under individual cavity tabs, illustrated in Fig. III-B. The information gathered on the cavity tabs includes settings for algorithm regulation accuracy, gain settings, divergence and motor constant thresholds as well as simultaneous quality factor and motor position change history plots.

\section{Ql Regulations Results at FLASH}

\section{A. Machine Condition During the Tool Operation}

The server performance test was conducted during regular FLASH accelerator operations and also during high-beam (4.5 mA) current tests, dedicated to the International Linear 


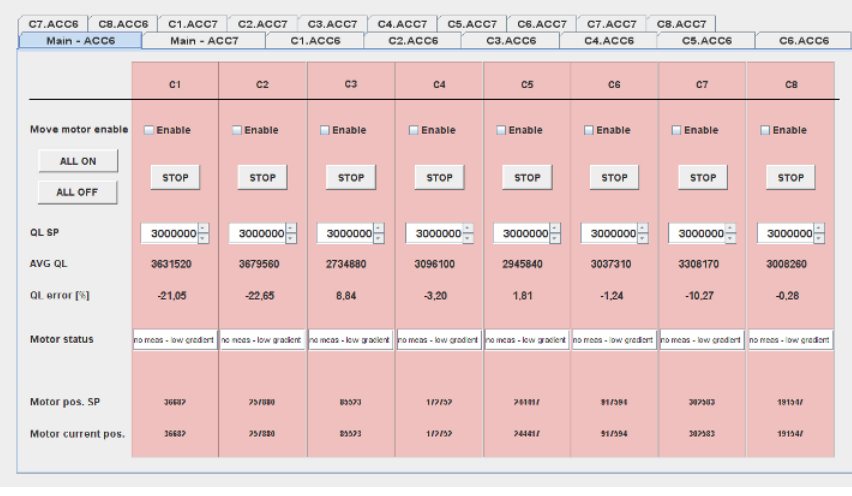

Fig. 7. Q1 adjustment tool main GUI panel

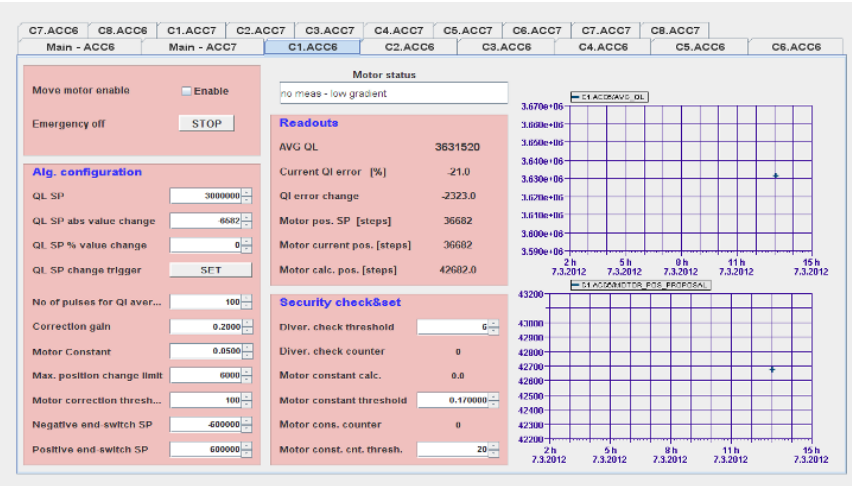

Fig. 8. Individual cavity GUI panel

Collider (ILC) 9mA tests [11], [10]. Over this test period, the application was extensively used for dynamic regulation of Q1 values to minimize the cavity field amplitude slopes.

\section{B. Results}

As previsouly emphasized, the two main driving reasons for Ql adjustments are:

1. compensation of thermal dependent Ql change, especially observed after switching the RF power off,

2. adjustment for different beam parameters operating conditions.

A couple of examples of transient behavior of cavity Q1 are represented in Fig. 9 and 10. Automatic Q1 adjustments to the nominal value of $3 \mathrm{e} 6$ have been efficiently achieved for all cavities in the controlled modules.

With the present LLRF system hardware at FLASH, the signal detection and acquisition system allowed for Ql settings with an accuracy of $\approx 2 e 5$ (for nominal $\mathrm{Ql}=3 \mathrm{e} 6$ ). This resolution meets the user requirements for $\mathrm{Ql}$ control precision, for the various beam current operations at FLASH. During one week operation with high beam current, no significant problems related to the algorithm work was encountered. Although the $\mathrm{Q} 1$ regulation was precise and reacted correctly to setting changes (feedback speed or regulation precision threshold for example), the only issue encountered is related to incorrect measurements of the quality factor due to cavity detuning. This caused an extensive motor excursion and finally, yielded to

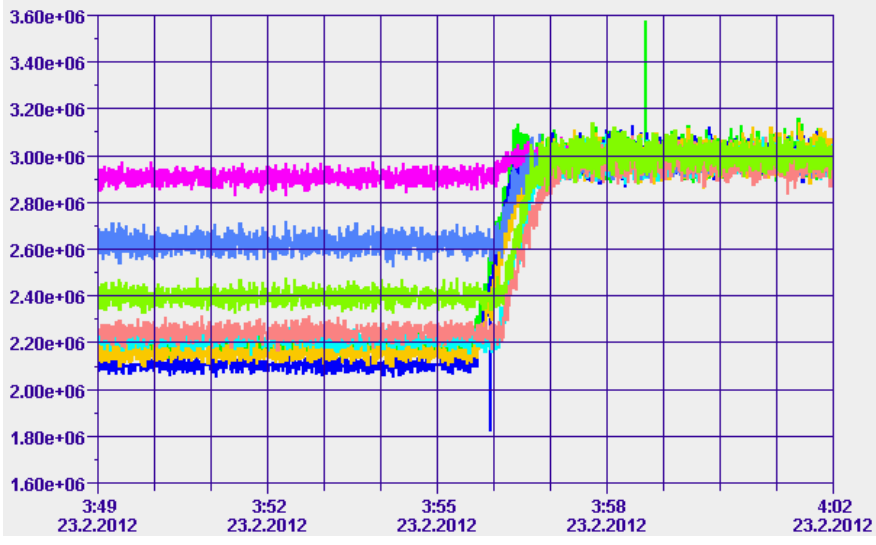

Fig. 9. Q1 automatic regulation for a single cryomodule (FLASH-ACC6)

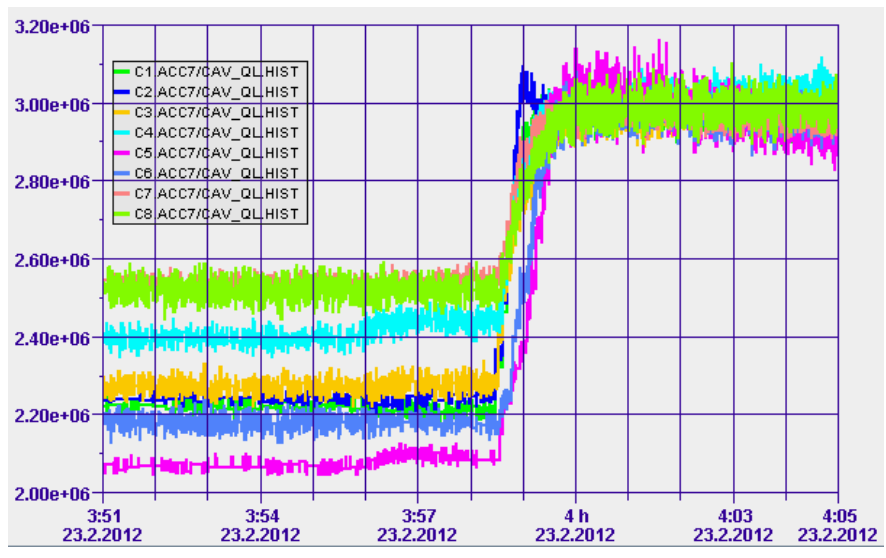

Fig. 10. Q1 automatic regulation for a single cryomodule (FLASH-ACC7)

algorithm termination without reaching the requested set-point value. Following this event, the restrictions for acceptable cavity gradient operations were refined in order to minimize future occurrences of this event.

\section{Ql Regulation Algorithm Improvements and Future Works}

The FEL facilities hosted by DESY (Hamburg) are now being equipped with new (uTCA platform based) LLRF systems hardware. The newly installed systems will not only improve the system availability but also provide higher resolution for the measured signals (the acquisition cards that are used in the system are equipped with 16 bit ADC's with up to $125 \mathrm{MHz}$ sampling frequency). These hardware upgrades will allow for a more precise cavity parameters identification, including Q1 and detuning. Tests of LLRF controllers equipped with the new hardware/software set-up are scheduled for summer of 2012. One main concern is to avoid over-exercising the Q1 motors, which is always a potential risk when applying any feedback control. Two safeguards are planed to be implemented for this purpose: first, the maximum motor number of motor steps per request is limited to a pre-defined threshold; second, the total motor excursion is monitored and logged.

\section{SUMmary AND CONCLUSION}

The loaded quality factors of individual cavities in the cryomodules at FLASH are automatically controlled by a slow 
feedback algorithm. This algorithm has been implemented as distributed software control system. Its performance has been evaluated and the overall system limitations were identified and quantified. The proposed Ql control gives an opportunity for a tighter control of the cavity field towards high beam loading operations (crucial for the ILC). It opens the way for new accelerator tuning strategies [10] and towards nearcontinuous wave operation scenarios which is one of the proposed upgrades for the European X-FEL.

\section{REFERENCES}

[1] K.Banes et. al., "RF Distribution Optimization in the Main Linacs of the ILC", PAC07 proceedings

[2] G. Cancelo et.al., "Analysis of DESY-FLASH LLRF Measurements for the ILC Heavy Beam Loading Test", PAC09 proceedings

[3] "The European X-Ray Free Electron Laser Technical Design Report", http://xfel.desy.de

[4] K. Przygoda, T. Pozniak, A. Napieralski, M. Grecki,"A novel approach for automatic control of piezoelectric elements used for Lorentz force detuning compensation", Mixdes 2009, 25-27 June 2009, p. 85-88

[5] T.Schilcher, "Vector Sum Control of Pulsed Accelerating Fields in Lorentz Force Detuned Superconducting Cavities", PhD Thesis.

[6] J.Branlard et. al, "LLRF Testing of Superconducting Cryomodules for the European XFEL", IPAC12, New Orleans, USA

[7] MicroTCA ${ }^{\circledR}$ is a trademark of PICMG, MTCA.4 specifications: http://www.picmg.org

[8] Reference for the Distributed Object Oriented Control System (DOOCS), http://doocs.desy.de

[9] Java DOOCS Data Display, http://jddd.desy.de/

[10] J. Carwardine et. al, "Report on the $2^{\text {nd }}$ Workshop on Linac Operations with Long Bunch-trains", ILC report 2011-032

[11] J. Branlard, "ILC high beam current tests at FLASH",2011FLASH Highlights 